\author{
¿Cómo citar este artículo? \\ Paredes Daza, J. D. y Pinto Linares, H. A. (septiembre-diciembre, 2019). La enseñanza de automatis- \\ mos eléctricos en el segundo nivel de educación media técnica o académica. Revista Virtual \\ Universidad Católica del Norte, (58), 139-161. doi: https://doi.org/10.35575/rvucn.n58a8
}

\title{
| La enseñanza de automatismos eléctricos en el segundo nivel de educación media técnica 0 académica ${ }^{1}$
}

The teaching of electrical automatisms in the second level of technical or academic middle education

\section{Javier David Paredes Daza}

Magister en Educación

Universidad Pedagógica y Tecnológica de Colombia

Colombia

javier.paredes@uptc.edu.co

Orcid: https://orcid.org/0000-0002-0336-9335

\section{Heriberto Augusto Pinto Linares}

Magíster en Ingeniería - Automatización Industrial Universidad Pedagógica y Tecnológica de Colombia Colombia

heriberto.pinto@uptc.edu.co

Recibido: 08 de mayo de 2018

Evaluado: 27 de marzo de 2019

Aprobado: 22 de julio de 2019

Tipo de artículo: Investigación científica y tecnológica.

\section{Resumen}

Con los resultados del presente artículo se pretende mejorar significativamente el proceso de enseñanza-aprendizaje de automatismos eléctricos, en algunos colegios técnicos o académicos de la ciudad de Duitama -Boyacá que lo requieren, y que además estos sean un referente pedagógico y didáctico para que otras instituciones educativas, en el ámbito nacional, propendan por su proliferación. El estudio está fundamentado metodológicamente desde la investigación documental y la investigación de enfoque cualitativo. Como parte de los resultados obtenidos se elaboraron cuatro tableros didácticos y nueve guías de práctica, que tienden a minimizar las dificultades tanto de docentes como de estudiantes en la enseñanza y aprendizaje, respectivamente, de los automatismos eléctricos, e incentivar a estos, a través del mejoramiento de la infraestructura de los laboratorios de electricidad. Los aportes del estudio realizado, además de beneficiar la construcción del conocimiento y el proyecto de vida de los educandos, contribuye a que estos futuros profesionales sean partícipes en proyectos que permitan el avance tecnológico del departamento y del país. Asimismo, el profesor, en su rol de guía en la enseñanza de automatismos eléctricos, cuenta con los elementos necesarios para crear un ambiente propicio que genere experiencias de aprendizaje significativo y con sentido.

Palabras clave: Automatización, Control automático, Enseñanza secundaria (2o nivel), Guía. 


\section{Abstract}

With the results of this article it is intended to improve in a significant way the teaching-learning process of electric automatisms, in some technical or academic schools in the city of Duitama Boyacá that require it, and that these be able to a pedagogical and didactic reference, so that other institutions educational programs at the national scope help to proliferate it. The study is methodologically based on documentary research and qualitative research. As part of the results obtained four didactic boards and nine practice guides were developed, which tend to minimize the difficulties of both teachers and students in the teaching and learning, respectively, of the electric automatisms and encourage them, through improvement of the infrastructure of the electricity laboratories. The contributions of the study carried out, in addition to benefiting the construction of knowledge and the life project of the students, contribute to these future professionals being participants in projects that allow the technological advance of the department and the country. Also, the teacher in his role as a guide in the teaching of electrical automatisms, has the necessary elements to create an enabling environment that generates significant and meaningful learning experiences.

Keywords: Automation, Automatic control, Secondary education (2nd level), Guidance.

\section{| Introducción}

La humanidad desde épocas inmemoriales ha desarrollado artefactos para aminorar el tiempo y esfuerzo dedicado a sus labores cotidianas. Por tanto, contaban con algunas máquinas que facilitaban sus actividades, otras (autómatas) eran usadas para entretener a las personas de la corte y servir de atracción en las festividades. De ahí que se considere a los sistemas de automatización, empleados en la industria, como los sucesores de los autómatas mecánicos del pasado.

En tal sentido, introducir modelos de automatización en los procesos industriales se ha convertido, con el transcurrir del tiempo, en un paso imprescindible para la evolución de los sectores de la economía del mundo, y en este escenario Colombia no es la excepción (Martínez, 2017). De este modo, Muñoz (2017) menciona:

La automatización llega en un momento en el cual las empresas buscan optimizar todos sus procesos. El reto entonces, será orientar las máquinas a aquellos procesos de carácter operativo y dejar los de naturaleza analítica a los humanos. Esto implica una mayor exigencia en las habilidades y conocimientos de las personas. Por consiguiente, las claves que se recomienda para enfrentar un mundo laboral cada vez más automatizado son: comprender el uso de la tecnología en un cargo; entender cómo integrar las operaciones con la automatización; fortalecer las habilidades analíticas para comprender datos arrojados por computadores; relacionarse con trabajadores de otras áreas; y, sobre todo, estar dispuesto al cambio. 
De ahí que profesionales de diferentes ámbitos del saber tecnológico, como ingenieros mecánicos, electrónicos e informáticos, sean los actores principales en la implementación de nuevas técnicas de fabricación y el empleo de diversos elementos tecnológicos que incluyen automatismos, permitiendo de esta manera que los procesos de producción, llevados a cabo en la industria, sean de alta calidad. "Sin embargo, también existen las demandas de las poblaciones jóvenes de contar con opciones de formación en esta especialidad” (Acuña, 2006, p. 2).

Así las cosas, hoy en día los jóvenes, a través de su formación en instituciones educativas de educación media académica o técnica, o mediante el Servicio Nacional de Aprendizaje -SENA(técnicos profesionales o tecnólogos), pueden ser partícipes en el diseño e implantación de proyectos que permitan un mejor desarrollo y avance de la tecnología en el país. Sin embargo, para los estudiantes de la especialidad de electricidad de algunos colegios técnicos o académicos de la ciudad de Duitama-Boyacá, esa posibilidad ha sido limitada, debido a que, actualmente, sus laboratorios no cuentan con tableros aptos para la realización de prácticas en automatismos eléctricos; por tanto, las diferencias entre teoría y práctica son evidentes, ya que el docente debe usar métodos tradicionales de aprendizaje memoristico para lograr que sus educandos visualicen cómo se interconectan los dispositivos en un sistema cableado, y asimilen los diversos conceptos que sólo se adquieren con la práctica.

Por consiguiente, con el desarrollo de la presente investigación, se pretende realizar un aporte significativo a los laboratorios de electricidad de las instituciones previamente mencionadas, mediante el diseño, construcción e implementación de tableros didácticos y sus respectivas guías de práctica; aporte que, además de contribuir a minimizar las dificultades de aprendizaje e incentivar a los estudiantes de grado once, mejorará la infraestructura de los laboratorios de electricidad y los procesos de enseñanza de los automatismos eléctricos, facilitando a los docentes del área nuevos recursos para favorecer el mejoramiento de sus actividades académicas y el cumplimiento de su función social.

A continuación, se presentan los referentes tomados para el diseño de las guías de práctica y el diseño de los tableros.

\section{Referentes para el diseño de las guías de práctica}

Las guías de práctica son un recurso que propicia la interacción entre el docente y el educando, y en consecuencia potencian el proceso de enseñanza y aprendizaje. Este tipo de recurso permite, entre otras cosas, proporcionarle al estudiante información relacionada con algún tipo de actividad que se pretenda este desarrolle. Así pues, apropiada dicha información, el alumno debe adquirir una serie de habilidades que le coadyuvan a llevar a feliz término el desarrollo de la tarea o procedimiento encomendado por el docente. 
En este orden de ideas, para el desarrollo de las guías de práctica de la presente investigación, se hace uso del modelo pedagógico conductista, ya que estas tienen como propósito orientar al estudiante hacia el logro de un objetivo, llevando el proceso formativo de modo sistemático, preciso y, de alguna manera, proyectando cómo se comportará el alumno durante y después del desarrollo de las guías en mención. Además, para dicho desarrollo se tuvieron en cuenta las estrategias pedagógicas de enseñanza preinstruccionales y coinstruccionales, porque además de preparar a los estudiantes respecto a la forma en que se va a aprender, se considera la conceptualización de contenidos y el mantenimiento de la atención y motivación, mediante el uso de ilustraciones y analogías (esquemas).

Por tanto, a través de la revisión de diversas referencias bibliográficas que abordan la elaboración de guías de práctica, se tuvieron en cuenta los siguientes referentes:

González (2007) realizó una investigación titulada “Auxiliar de servicios técnicos del laboratorio de física de la Universidad Pedagógica y Tecnológica de Colombia (UPTC), Sede Duitama y realización de guías de laboratorio para controles eléctricos", con el objetivo de desempeñar la labor de auxiliar de servicios técnicos del laboratorio de física en los talleres de la UPTC, Sede Duitama, complementándola con la elaboración de guías para los laboratorios de controles eléctricos. Esta guía adhiere los siguientes componentes: título, introducción, objetivos, generalidades, materiales y herramientas, procedimiento, cuestionario.

Asimismo, Albarracín y Granados (2003) realizaron una investigación titulada “Implementación del área de controles y accionamientos eléctricos en el Instituto Nacional de Promoción Social de Duitama", con el objetivo de proponer un nuevo esquema en el área de controles y accionamientos eléctricos del Instituto Nacional de Promoción Social, cumpliendo con las normas requeridas en la Ley General de Educación, y ofreciendo a los futuros bachilleres una mejor posibilidad de desempeño laboral y/o académico. Se busca también, contribuir con el desarrollo de la educación en la región, teniendo en cuenta los avances científicos y tecnológicos que se presentan cada día para mejorar el nivel educativo de quienes los puedan estudiar y aplicar de manera útil. Esta guía consolida los siguientes componentes: código, número de práctica, título, objetivos, equipos y materiales, diagramas, funcionamiento, procedimiento, informe de práctica, evaluación.

\section{Referentes para el diseño de los tableros}

Para el diseño y posterior construcción de los tableros didácticos se tuvieron en cuenta los siguientes referentes:

Tablero didáctico UPTC (2008), presentado en la figura 1. Se encuentra en el laboratorio de física de la Universidad Pedagógica y Tecnológica de Colombia, Facultad Sede Duitama. 


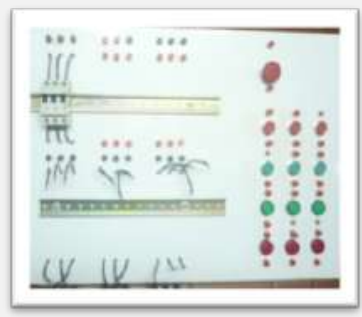

Figura 1. Tablero didáctico de automatismos eléctricos. Tomado del Laboratorio de física, UPTC Sede Duitama (2008).

Este tablero didáctico cuenta con pulsadores de marcha, pulsadores de parada, pulsador de emergencia, pilotos luminosos (verdes), pilotos luminosos (rojos), rieles omega, borneras, terminales para cable y automático. En este se pueden montar hasta tres etapas (contactores) y otros dispositivos, como temporizadores y contactores auxiliares; además, posee un recubrimiento en fórmica, lo cual facilita ubicar observaciones, índices y marcas de cada uno de los dispositivos.

Tablero didáctico SENA (2005), presentado en la figura 2. Se encuentra en el taller del SENA Seccional Sogamoso.

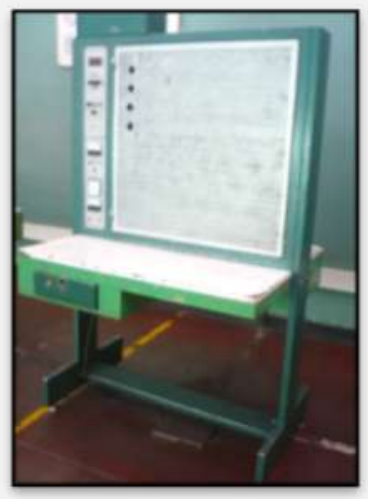

Figura 2. Tablero didáctico de automatismos eléctricos. Tomado de SENA Centro multisectorial Sogamoso (2005). 
En la parte izquierda el tablero en mención tiene una lámina en la cual se fija el seccionador mediante tornillos; también, cuenta con unas borneras R, S, T (sirven para alimentar el circuito), una malla que posee cuatro huecos en la parte superior (alli se colocan los pulsadores o pilotos luminosos), un riel omega (se asegura con tornillos a la malla y sirve para fijar otros dispositivos), y un cajón en el cual se guardan algunos materiales y herramientas que se necesitan para la práctica.

\section{Metodología}

La metodología adoptada para el desarrollo de la presente investigación, se muestra a continuación (véase figura 3).

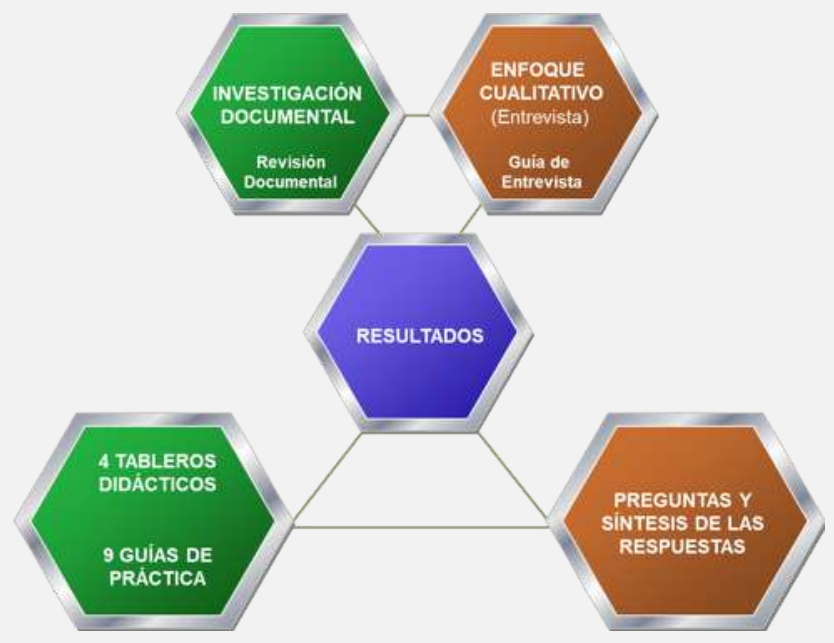

Figura 3. Proceso metodológico. Elaboración propia.

En ese sentido, se puede apreciar que el estudio se abordó desde una metodología de investigación documental, mediante la cual se recopiló la información que permitiera el diseño, construcción e implementación de los tableros didácticos y sus respectivas guías de práctica, para la enseñanza de automatismos eléctricos en el segundo nivel de la educación media técnica o académica. Así las cosas, Gómez (2011) manifiesta que, 
La investigación documental tiene un carácter particular de dónde le viene su consideración interpretativa. Intenta leer y otorgar sentido a unos documentos que fueron escritos con una intención distinta a esta dentro de la cual se intenta comprenderlos. Procura sistematizar y dar a conocer un conocimiento producido con anterioridad al que se intenta construir ahora. (p. 230).

Así pues, la técnica usada fue la revisión documental, la cual permitió explorar, seleccionar, sintetizar y analizar la información consultada en los documentos. Por consiguiente, para la recolección de información se utilizaron los siguientes instrumentos: fichas bibliográficas (se recopilaron los datos de las diferentes fuentes), fichas textuales (para tomar la información más importante de las fuentes consultadas), fichas de resumen (para sintetizar los textos y documentos con algunas opiniones personales) y fichas de análisis (para describir y confrontar la información suministrada por los autores consultados). Cabe mencionar que la selección de material óptimo dentro del desarrollo de las guías de práctica, se realizó teniendo en cuenta los contenidos programáticos de la asignatura de Automatismos Eléctricos de grado once, y mediante la asesoría de los docentes del área eléctrica de los cuatro colegios de la ciudad de Duitama-Boyacá (se identificaron las temáticas mínimas e indispensables a desarrollar por medio de las guías de práctica).

Además, se empleó la investigación de enfoque cualitativo, buscando conocer de primera mano la experiencia vivida desde el punto de vista de las personas que la perciben; de esta manera, se usó como técnica la entrevista, la cual fue aplicada a los docentes del área eléctrica de algunos colegios técnicos o académicos de la ciudad de Duitama-Boyacá, luego de hacer uso de las guías de práctica y los tableros didácticos. Así pues, como instrumento para la recolección de la información, se hizo uso de la guía de entrevista, la cual se encuentra conformada por siete preguntas, que pretenden vislumbrar la opinión de los profesores sobre los resultados obtenidos en la presente investigación. Luego de la aplicación de la entrevista, se procedió a realizar el análisis de la información recolectada y, de esta manera, determinar el impacto de los tableros didácticos y sus respectivas guías de práctica en la enseñanza de automatismos eléctricos en el segundo nivel de la educación media técnica o académica.

Por otra parte, cabe señalar que de las 14 instituciones educativas públicas de carácter técnico o académico con las que cuenta la ciudad de Duitama, se tomó una muestra de cuatro instituciones (tres de carácter técnico y una de carácter académico). Para su elección se consideraron los siguientes criterios: las difíciles condiciones que presentan sus laboratorios de electricidad, lo cual ha generado dificultades en el proceso de enseñanza y aprendizaje de los automatismos eléctricos; que el plantel cuente con la especialidad en electricidad y que las directivas de los colegios contribuyan con la compra de los materiales y dispositivos necesarios para la elaboración e implementación de los tableros didácticos. 


\section{| Resultados}

En primera instancia, y en pro de dar cuenta de la revisión documental propuesta en la metodología, a continuación, se presenta la información obtenida a través de sus instrumentos. Por consiguiente, mediante las fichas bibliográficas se elaboró un listado de las diferentes fuentes que abordan la temática de los automatismos eléctricos, especialmente sobre aquellas enfocadas en el diseño y elaboración de tableros didácticos y guías de práctica. Así pues, dentro de esta exploración se encontraron los siguientes referentes:

González y Macías (2013): Tablero de control eléctrico; Álvarez y Araque (2004): Diseño y construcción de un banco para controles y automatismos eléctricos con 10 guías de trabajo para el taller de electricidad del Instituto Educativo Técnico diversificado de Monterrey-Casanare; Arcos y Chicaiza (2015): Diseño y construcción de un tablero de control automático para la corrección del factor de potencia, empleando un módulo DCRA; González (2007): Auxiliar de servicios técnicos del laboratorio de física de la Universidad Pedagógica y Tecnológica de Colombia (UPTC); Albarracín y Granados (2003): Implementación del área de controles y accionamientos eléctricos en el Instituto Nacional de Promoción Social de Duitama; el tablero didáctico UPTC (2008) y el tablero didáctico SENA (2005); los contenidos programáticos de la asignatura de Automatismos Eléctricos de grado once; entre otros.

Ahora bien, al tener el listado de las fuentes bibliográficas, se procedió por medio de las fichas textuales a tomar de cada una de estas la información de mayor importancia, logrando evidenciar durante este ejercicio que gran parte de los referentes no cumplían con las expectativas de los autores. Seguidamente, con el uso de las fichas de resumen se sintetizó la información de los recursos seleccionados, de tal manera que se tuviera claridad sobre los componentes de la guía de práctica, y los materiales y dispositivos del tablero didáctico, aportados por cada uno de ellos.

Finalmente, a través de las fichas de análisis se realizó la confrontación de la información sintetizada, evidenciándose cierta similitud entre algunas investigaciones. En virtud de ello, los referentes en los cuales se basó la presente investigación, fueron los siguientes: González (2007), Albarracín y Granados (2003), y los contenidos programáticos de la asignatura de Automatismos Eléctricos de grado once. Estos sirvieron como insumo para la obtención de los componentes propuestos, en pro de elaborar las guías de práctica; mientras que, el tablero didáctico UPTC (2008) y el tablero didáctico SENA (2005) fueron de gran aporte para la construcción de los tableros didácticos.

En ese orden de ideas, a continuación, se da muestra tanto de los componentes propuestos para la elaboración de las guías de práctica (con su respectiva descripción), como de los materiales y dispositivos seleccionados para la elaboración y funcionamiento de los tableros didácticos (ver tablas 1 y 2 ). 
Tabla 1

Componentes de la guía de práctica

\begin{tabular}{|c|c|}
\hline Componentes & Descripción \\
\hline Título & Se enuncia el número de la guía de práctica y el nombre de la institución. \\
\hline Capítulos & $\begin{array}{l}\text { Se da a conocer el título de la guía de práctica, la asignatura, el tiempo } \\
\text { dispuesto para el desarrollo de la práctica y el lugar donde se desarrollará. }\end{array}$ \\
\hline & Mediante el objetivo general y los objetivos específicos se definen los logros \\
\hline Objetivos & que el estudiante debe alcanzar. \\
\hline Conve & $\begin{array}{l}\text { Se muestran los dispositivos que se van a utilizar para el desarrollo de la guía } \\
\text { de práctica. Incluye la marca, el símbolo del esquema de mando, fuerza y una } \\
\text { foto. }\end{array}$ \\
\hline Simulación & Se le propone al estudiante que simule los esquemas de mando y fuerza. \\
\hline Esquemas & Se disponen los esquemas de mando y fuerza. \\
\hline $\begin{array}{l}\text { Ciclo de } \\
\text { funcionamiento }\end{array}$ & $\begin{array}{l}\text { Se enuncia paso a paso lo que ocurre con los dispositivos y se explica el } \\
\text { funcionamiento del circuito. }\end{array}$ \\
\hline $\begin{array}{l}\text { Esquema de } \\
\text { interconexiones }\end{array}$ & $\begin{array}{l}\text { Basado en el esquema de mando, se analizan y conectan gráficamente los } \\
\text { dispositivos de mando, maniobra, señalización y protección, permitiendo } \\
\text { realizar las conexiones en el tablero con mayor seguridad y certeza. }\end{array}$ \\
\hline $\begin{array}{l}\text { Materiales y } \\
\text { herramientas }\end{array}$ & $\begin{array}{l}\text { Se presenta la lista de materiales y herramientas necesarios para la realización } \\
\text { de la práctica. }\end{array}$ \\
\hline Montaje & $\begin{array}{l}\text { Se enuncia detalladamente el conjunto de actividades necesarias para realizar } \\
\text { la práctica. }\end{array}$ \\
\hline Cuestionario & $\begin{array}{l}\text { Se disponen preguntas referentes a la práctica como de la etapa de realización } \\
\text { y de los contenidos temáticos del área. }\end{array}$ \\
\hline $\begin{array}{l}\text { Informe guía } \\
\text { de practica }\end{array}$ & $\begin{array}{l}\text { Se mencionan algunos lineamientos que se deben tener en cuenta para } \\
\text { entregar el informe al docente del área. }\end{array}$ \\
\hline Observaciones & $\begin{array}{l}\text { Se consignan algunas recomendaciones que el estudiante debe tener en cuenta } \\
\text { para la realización de futuras prácticas. }\end{array}$ \\
\hline Bibliografia & $\begin{array}{l}\text { Se muestran las referencias bibliográficas utilizadas para la consulta posterior } \\
\text { a la práctica. }\end{array}$ \\
\hline Infografía & $\begin{array}{l}\text { Se presentan las referencias electrónicas utilizadas para la consulta posterior } \\
\text { a la práctica. }\end{array}$ \\
\hline
\end{tabular}

Nota: Elaboración propia. 
Tabla 2

Materiales y dispositivos para la elaboración e implementación de los cuatro tableros didácticos

\begin{tabular}{cl}
\hline Cantidad & \multicolumn{1}{c}{ Concepto } \\
\hline 4 & Tablas de 75cm*85cm \\
8 & Pulsadores verdes \\
4 & Pulsadores rojos \\
4 & Pulsadores de emergencia \\
8 & Seccionador trifásico \\
8 & Pilotos luminosos verdes \\
20 & Pilotos luminosos rojos \\
36 & Borneras o regletas de 4P \\
1 & Borneras o regletas de 2P \\
1.20 metros & Lamina de fórmica 1.20*2.40 \\
50 & Soporte tablero (quíntuples) \\
20 & Terminales para cable \\
& Metros de cable N-12 AWG color \\
1.20 metros & blanco \\
\end{tabular}

Nota: Elaboración propia.

Posteriormente, se da a conocer la entrevista grupal (desarrollada mediante la investigación de enfoque cualitativo), realizada a ocho profesores del área eléctrica de las instituciones educativas tomadas en la muestra, con el ánimo de identificar las dificultades y fortalezas emanadas del uso de los tableros didácticos y las guías de práctica, resultado de esta investigación. En la tabla 3 se exponen las preguntas realizadas y la síntesis de las respuestas derivadas de dicha entrevista. 
Tabla 3

Guia de entrevista - preguntas y sintesis de las respuestas

\begin{tabular}{|c|c|}
\hline $\mathrm{N}^{\circ}$ & Preguntas \\
\hline 1 & $\begin{array}{l}\text { ¿Cuál es su apreciación sobre la } \\
\text { forma como se conectan los } \\
\text { dispositivos en el tablero, de } \\
\text { acuerdo al esquema de mando? }\end{array}$ \\
\hline 2 & $\begin{array}{l}\text { ¿El tablero didáctico cumple con } \\
\text { normas de seguridad? }\end{array}$ \\
\hline
\end{tabular}

Los docentes, en común acuerdo, manifestaron que, aunque les parece acertada la conexión de los dispositivos en el tablero a través del esquema de mando, al estudiante inicialmente le ha sido complejo realizarla mediante dicho esquema.

Aseguraron, sin excepción alguna, que los tableros didácticos indudablemente cuentan con las normas básicas de seguridad, previniendo accidentes y promoviendo el cuidado del material de los laboratorios.

Para el desarrollo de las guías, se hace necesario que el estudiante 3 complemente su práctica con el desconocimiento ante el programa Automation software Automation Studio, Studio y sobre cualquier otro que propenda por la simulando lo realizado ¿Sabe simulación de automatismos eléctricos. usarlo?

Aunque su respuesta fue negativa, resaltaron unánimemente la importancia que tiene el esquema

Teniendo presente que a partir del esquema de mando se obtiene el esquema de interconexiones $¿$ sabe elaborarlo? de interconexiones para el fácil desarrollo de las guías de práctica. Además, dieron a conocer la necesidad de recibir orientación acerca de la elaboración de dicho esquema, con el propósito de facilitarle al educando un recurso que le permita, poco a poco, hacer una compresión más certera del esquema de mando.

¿Qué opina sobre la funcionalidad y Aseguraron que los tableros didácticos son diseño de los tableros didácticos? funcionales, dado que satisfacen las necesidades 
6

De acuerdo a las guías de práctica, ¿qué piensa sobre su evolución, impacto visual, organización y didáctica?

7

¿Los resultados emanados del presente estudio qué opinión le merecen como aporte a la educación? para las que fueron creados. Seguidamente, en cuanto al diseño expresaron que los tableros en mención consienten un trabajo armónico y son de fácil movilidad.

Coincidieron en que las guías de práctica son innovadoras, ya que sus imágenes, esquemas, estructura y aporte didáctico se complementan, generando en el estudiante un impacto visual que lo estimula ampliamente en su proceso de aprendizaje. Todos los docentes manifestaron que el aporte de la presente investigación favorece, sin duda alguna, el proceso de enseñanza y aprendizaje de automatismos eléctricos, llevado a cabo en las instituciones educativas de carácter técnico o académico, en al contexto nacional e internacional. En ese sentido, fue catalogado como una contribución pedagógica y didáctica de gran significado para la educación.

Nota: Elaboración propia.

Luego de dar a conocer las apreciaciones de los docentes, respecto a cada una de las preguntas realizadas en la entrevista, se da paso a la discusión de los resultados, donde, entre otras cosas, se abordan las respuestas que requirieron de una solución por parte de los autores de la presente investigación.

\section{| Discusión}

En consonancia con los resultados arrojados por la revisión documental, vale la pena resaltar que a través de la exploración efectuada sobre estudios que abordan la temática de los automatismos eléctricos, especialmente sobre aquellos enfocados en el diseño y elaboración de tableros didácticos y guías de práctica, se logró evidenciar que son pocas las investigaciones al respecto. Además, es de anotar que de estos estudios tan sólo se tomaron como referencia una mínima parte, debido a que los restantes tenían similitud o no aportaban mayor información a la investigación.

En este sentido, a continuación, se describen en la tabla 4 los estudios afines, el valor agregado de la presente investigación y el aporte de los investigadores. 
Tabla 4

Contraste entre los resultados obtenidos y los resultados de otros estudios afines

\begin{tabular}{ccc}
\hline $\begin{array}{c}\text { Estudios afines } \\
\text { (Referentes) }\end{array}$ & $\begin{array}{c}\text { Valor agregado de la } \\
\text { investigación }\end{array}$ & $\begin{array}{c}\text { Aportes de los } \\
\text { investigadores }\end{array}$ \\
\hline
\end{tabular}

Auxiliar de servicios

técnicos del laboratorio

de física de la

Universidad Pedagógica

y Tecnológica de

Se incluyó el tiempo aproximado,

Colombia - UPTC.

(González, 2007)

Implementación del área de controles y

accionamientos

eléctricos en el Instituto

Nacional de Promoción

Social de Duitama.

(Albarracín y Granados, 2003)

Tablero didáctico de automatismos eléctricos.

(UPTC, 2008)

Tablero didáctico.

(SENA, 2005) el lugar, bibliografia e infografia, se mejoró la presentación de los esquemas, se incluyeron los símbolos, índices, marca, figura o foto (para el esquema de mando y de fuerza), ayudas didácticas como el software Automation Studio, un esquema de interconexiones y un cuestionario.

Dado que el tablero didáctico utilizado en el laboratorio de fisica de la UPTC, fue el principal referente, se corrigió la problemática de las borneras que se sustituyeron por una regleta que permite hacer conexiones seguras, lo que reduce significativamente el riesgo eléctrico.
Nueve guías de práctica (1.

Mando por impulso permanente; 2 . Mando por impulso inicial; 3. Mando por impulso inicial y permanente; 4. Mando por impulso inicial desde dos estaciones; 5 . Secuencia manual LIFO de dos etapas; 6. Secuencia automática LIFO de dos etapas; 7. Inversor manual de marcha; 8. Inversor automático de marcha controlado por un temporizador; y 9. Arrancador estrella triángulo controlado por un temporizador).

Cuatro tableros didácticos para el desarrollo de prácticas en automatismos eléctricos. 
Aunque el diseño de la guía realizado por González (2007) es apropiado, puede ser causal de las siguientes mejoras: optimar la presentación general de la guía, de manera que se cree un mejor efecto visual; incluir el tiempo aproximado y el lugar donde se desarrollará la práctica; incluir bibliografía e infografía para consultas posteriores; optimar la presentación de los esquemas, de forma que sean mucho más claros y se vean más ordenados; sugerir alguna ayuda didáctica para simular los esquemas de mando y fuerza; incluir recursos educativos para ayudar a los estudiantes a comprender cómo se interconectan los dispositivos de mando, maniobra, señalización y protección antes de realizar las prácticas en vivo y en directo; describirle con claridad al estudiante, los elementos y condiciones en que debe entregar su informe de práctica.

Asimismo, y pese a que el diseño de la guía elaborada por Albarracín y Granados (2003) cumple su propósito, además de las mejoras expuestas en el anterior referente, se sugieren las siguientes: incluir el nombre de la asignatura; optimar la presentación de la tabla de equipos y materiales, ya que se debería incluir el símbolo, los índices, la marca y una foto; e incluir un cuestionario sobre temáticas relacionadas con la práctica, tanto de la etapa de realización como de los contenidos temáticos del área.

Seguidamente, para el diseño y elaboración de las guías de práctica, se tuvieron en cuenta los contenidos programáticos de la asignatura de Automatismos Eléctricos de grado once, en donde se destacan: las clases de esquemas, los símbolos de automatismos eléctricos, los dispositivos empleados en accionamientos eléctricos, los elementos de señalización, mando de un motor por impulso permanente, mando de un motor por impulso inicial, mando de un motor por impulso inicial y permanente, mando por impulso inicial y permanente desde dos estaciones, arranque directo de un motor, tipos de temporizadores e inversor de giro. Así pues, con la asesoría de los docentes del área eléctrica de los cuatro colegios de la ciudad de Duitama-Boyacá, se identificaron las temáticas mínimas e indispensables a desarrollar por medio de las nueve guías de práctica (véase apéndice 2 ) $^{2}$ elaboradas en pro de la formación de los estudiantes. Dichas temáticas se encuentran en la tabla 4, específicamente en la columna relacionada con los aportes de los investigadores.

Ahora bien, para el diseño y elaboración de las guías de práctica, además de lo previamente mencionado, se tuvo en cuenta lo siguiente:

Para mejorar la presentación general de la guía, se separó cada una de sus secciones a través de una barra rectangular, en la cual aparece el título de la misma; se adhirió el tiempo y el sitio donde se realizará la práctica; se incluyeron las referencias bibliográficas; la presentación de los esquemas fue mejorada; la tabla de convenciones tiende a ser más clara, se incluyeron los símbolos tanto para el esquema de mando como para el de fuerza, sus correspondientes indices, marca, figura o foto; se adicionaron actividades de simulación por medio del software Automation Studio; como recurso educativo y didáctico se elaboró un esquema de interconexiones que le ayuda al estudiante a comprender cómo se interconectan cada uno de los dispositivos; en pro de favorecer el desarrollo exitoso de las prácticas a realizar, se adicionó un cuestionario y, por último, se mostraron los parámetros necesarios para la entrega del respectivo informe de práctica.

2 Como ejemplo se presenta la guía de práctica № 1. 
Cabe mencionar que el desarrollo de prácticas de laboratorio, mediante el uso de guías bien estructuradas, conlleva a que el estudiante entienda la teoría. Rodríguez (s.f.) menciona que,

(...) la práctica sin teoría es un salto al vacio, la teoría dirige la práctica de un modo ordenado y sistemático, evitando improvisaciones, y la práctica a su vez, muestra los obstáculos encontrados, los logros, los imprevistos, etcétera, en interacción constante. (p. 3).

Sin duda, la práctica sin teoría o la teoría sin práctica, desfavorecen considerablemente el desarrollo de las actividades académicas, generando diversas dificultades en el desempeño del educando. En palabras de Alemán y Mata (2006):

Es necesario tener presente que las prácticas de laboratorio deben de estar coordinadas con las clases de teoría. Sin embargo, varias circunstancias hacen que esto no siempre sea posible a causa de la distribución horaria, el número de horas disponibles para el laboratorio, número de alumnos y la disponibilidad económica para comprar suficientes equipos y material.

Por otra parte, y teniendo en cuenta los referentes para la elaboración de los tableros didácticos, cabe señalar que aunque el diseño y la distribución de los dispositivos del tablero didáctico UPTC (2008) se consideran adecuados, y el tamaño del tablero es apropiado, ya que se puede trasladar con facilidad y almacenarse en un reducido espacio, resulta particularmente problemático realizar conexiones seguras, debido a que las borneras no sujetan firmemente los conductores, quedando expuestos a un posible contacto (alto riesgo eléctrico) (Véase figura 1).

Del mismo modo, a pesar de que el tablero didáctico SENA (2005) cuenta por cada lado con un espacio de trabajo (permite que puedan trabajar paralelamente dos grupos de estudiantes), este es muy grande (requiere mucho espacio), está fijo al piso (impide la movilidad de los estudiantes), y su verticalidad lo hace poco ergonómico y visualmente inadecuado; además, los estudiantes deben montar y desmontar los dispositivos en el tablero, lo que podría hacer que se dañen con rapidez (Véase figura 2). 
En ese sentido, es de anotar que, como referente principal para la construcción de los cuatro tableros didácticos, en pro del desarrollo de las prácticas en automatismos eléctricos, se tuvo en cuenta el tablero empleado en el laboratorio de física de la Universidad Pedagógica y Tecnológica de Colombia, corrigiéndose la problemática de las borneras que se sustituyeron por una regleta que permite hacer conexiones seguras, lo que reduce significativamente el riesgo eléctrico. Por tanto, en el apéndice 1 se presenta y describe el tablero, resultado de la presente investigación, el cual se acomoda a las necesidades de los cuatro colegios considerados en la muestra. Cabe destacar que, a partir del esquema de interconexiones más complejo de las guías de práctica (Secuencia manual LIFO de dos etapas), se estableció el número de etapas y, por tanto, el número de dispositivos a montar sobre los tableros. Seguidamente, se ahonda en las respuestas generadas por los docentes a través de la entrevista grupal, primando aquellas que requirieron de una solución (véase tabla 3).

En ese orden de ideas, se aborda, en primera instancia, la preocupación de los profesores respecto a la dificultad que han tenido sus estudiantes a la hora de realizar la conexión de los dispositivos en el tablero, a través del esquema de mando. Tal dificultad de los educandos es superada a través del esquema de interconexiones, el cual se encuentra adherido a la guía de práctica y se encarga de ilustrar claramente las diferentes conexiones a realizar en el tablero didáctico. Además, se estableció un horario de capacitación para que los docentes aprendieran a elaborar el esquema de interconexiones y de esta manera asegurar que el aprendizaje del estudiante sea satisfactorio.

Finalmente, y en vista de que el conocimiento sobre los programas que simulan automatismos eléctricos era escaso en la mayoría de los docentes, se les proporcionó capacitación en el manejo del software Automation Studio, que "es un programa de concepción, animación y simulación. Ha sido creado para responder a las necesidades de ingeniería, formación y concepción en el campo de la automatización. Sus talleres reflejan lo más fielmente posible la realidad industrial" (Famic Technologies Inc., 2010, p. 1).

\section{| Conclusiones}

Aunque por medio de la revisión documental se hallaron varias investigaciones sobre la construcción de tableros didácticos y la elaboración de guías de práctica, centradas en la enseñanza de automatismos eléctricos, fueron pocos los referentes adheridos al presente estudio, debido a que la información aportada se tornaba repetitiva o no cumplía con las expectativas de esta investigación.

El diseño y posterior construcción de los tableros didácticos de automatismos eléctricos y sus respectivas guías de práctica, contribuyó notablemente al mejoramiento del proceso de enseñanza y aprendizaje llevado a cabo en las instituciones educativas afectadas, dado que actualmente cuentan con los recursos adecuados para que el docente logre generar en el aula un ambiente de aprendizaje que induzca a la construcción del conocimiento y que permita una mayor preparación académica y técnica de los estudiantes. 
Si bien, los resultados obtenidos en la presente investigación provocaron un cambio significativo en la forma como se impartían las clases de Automatismos Eléctricos en el segundo nivel de la educación media técnica o académica en algunos colegios de la ciudad de Duitama, es importante que estos recursos se den a conocer para que otras instituciones educativas, que están atravesando por dificultades semejantes, puedan acceder a ellos y, de esta manera, contribuyan a que sus educandos sean partícipes en proyectos que permitan el avance tecnológico del departamento y del país.

Cabe mencionar que la estructura de las guías de práctica incluye componentes que facilitan el aprendizaje del educando, permitiéndole desenvolverse satisfactoriamente en el desarrollo de las tareas propuestas; es decir, lo orientan acertadamente en el cumplimiento de sus objetivos. De esta manera, se considera que las guías en mención, además de ser un aporte pedagógico y didáctico, propenden por el trabajo en grupo en un ambiente colaborativo y participativo.

Vale la pena resaltar la importancia que tiene el esquema de interconexiones en el desarrollo de las guías de práctica, dado que es una puesta en escena que le posibilita al educando realizar en su casa la conexión gráfica de los dispositivos de mando, maniobra, señalización y protección, sin el impedimento que conlleva el no tener a la mano la infraestructura necesaria para tal fin. De esta manera, el estudiante logra tener mayor seguridad a la hora de efectuar las conexiones en el tablero y, entre otras cosas, aprende a identificar la marca, el símbolo del esquema de mando y fuerza de cada uno de los dispositivos utilizados.

Los resultados que arrojó la presente investigación favorecen la construcción del proyecto de vida del educando, permitiendo que este se desempeñe como un individuo competente en cada una de las tareas que emana el estudio de los automatismos eléctricos.

\section{| Referencias}

Acuña, A. L. (2006). Proyectos de robótica educativa: motores para innovación. Recuperado de http://www.fod.ac.cr/robotica/descargas/roboteca/articulos/2009/motorinnova_corto.pdf

Albarracín, N. y Granado, F. (2003). Implementación del área de controles y accionamientos eléctricos en el Instituto Nacional de Promoción Social (Trabajo de grado de pregrado). Universidad Pedagógica y Tecnológica de Colombia. Duitama, Colombia.

Alemán, J. y Mata, M. (2006). Guía de elaboración de un manual de prácticas de laboratorio, taller o campo: asignaturas teórico prácticas. México: Universidad Autónoma Chapingo. Recuperado de http://www.rivasdaniel.com/Pdfs/GUIAMANUALPRACTICAS.pdf

Álvarez, H. y Araque, L. (2004). Diseño y construcción de un banco para controles y automatismos eléctricos con 10 guías de trabajo para el taller de electricidad del Instituto Educativo Técnico diversificado de Monterrey-Casanare (Tesis Trabajo de grado de pregrado). Universidad Pedagógica y Tecnológica de Colombia. Duitama, Colombia. 
Arcos, E. y Chicaiza, D. (2015). Diseño y construcción de un tablero de control automático para la corrección del factor de potencia, empleando un módulo DCRA (Trabajo de grado de tecnología). Escuela Politécnica Nacional, Ecuador.

Famic Technologies Inc. (2010). Guía del usuario de Automation Studio. Recuperado de http://personal.biada.org/ lescudero/varis/Tutoriales/AS5_GUI_SF01_003.pdf

Gómez, L. (2011). Un espacio para la investigación documental. Revista Vanguardia Psicológica Clínica Teórica Y Práctica, 1(2), 226-233. Recuperado de https://dialnet.unirioja.es/descarga/articulo/4815129.pdf

González, N. (2007). Auxiliar de servicios técnicos de laboratorio de física de la Universidad Pedagógica y Tecnológica de Colombia, Facultad Seccional Duitama y realización de guías de laboratorio para controles eléctricos (Trabajo de grado de pregrado). Universidad Pedagógica y Tecnológica de Colombia. Duitama, Colombia.

González, L. y Macías, K. (2013). Tablero de control eléctrico (Trabajo de grado de pregrado). INSTITUTO POLITÉCNICO NACIONAL, México. Recuperado de http://www.academico.cecyt7.ipn.mx/Material/Trabajos_Investigadores_2013/TableroControlElectrico-TESIS.pdf

Martínez, J. (junio 12 de 2017). Automatización: una necesidad para Colombia. Vanguardia. Recuperado de http://www.vanguardia.com/mundo/tecnologia/400493-automatizacion-una-necesidad-para-colombia

Muñoz, E. (2017). La Automatización en Colombia. Recuperado de http://www.usergioarboleda.edu.co/escuela-de-negocios-prime/MBA/la-automatizacion-en-colombia/

Rodríguez, F. (s.f.). Ingeniería, fabricación y montaje de estructuras metálicas. Recuperado de www.obrapropia.com/viewinteriorobra.ashx?idObra=1163

Servicio Nacional de Aprendizaje. (2005). Tablero didáctico de automatismos eléctricos [Figura]. Sogamoso, Colombia.

Universidad Pedagógica y Tecnológica de Colombia. (2008). Tablero didáctico de automatismos eléctricos [Figura]. Duitama, Colombia

Apéndice 1.

Tablero didáctico para la enseñanza de automatismos eléctricos en el segundo nivel de la educación media técnica o académica. Elaboración propia. 


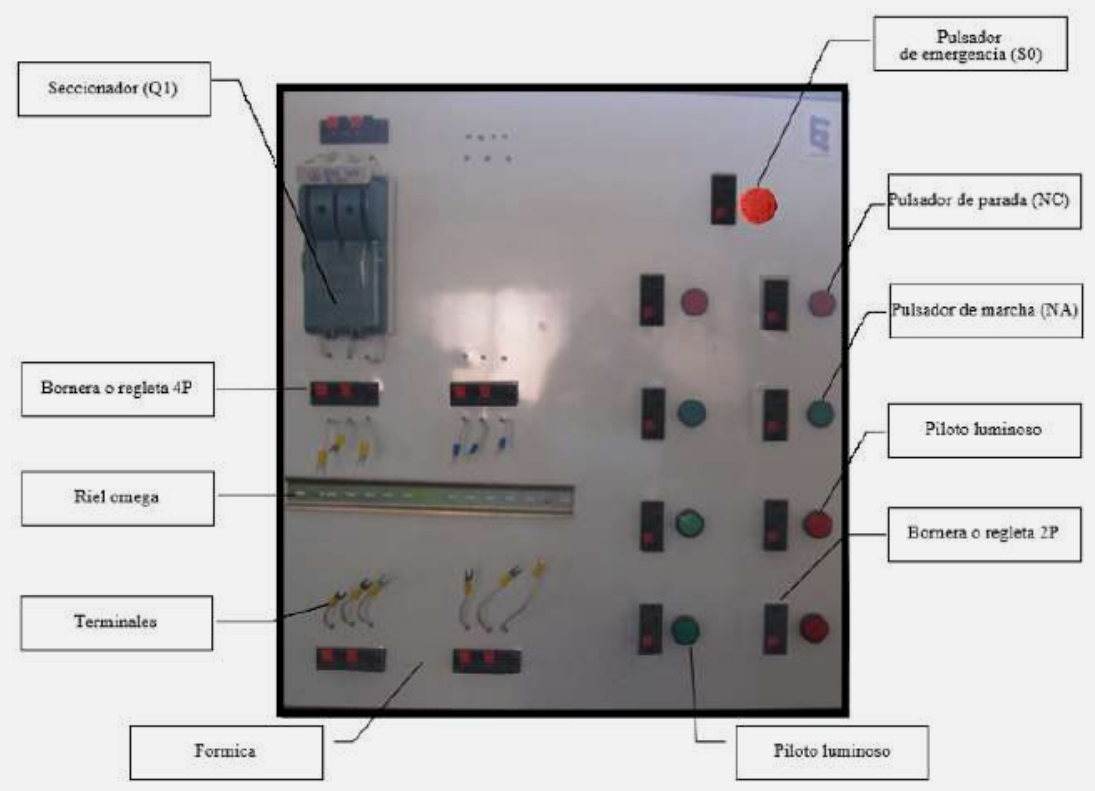

Apéndice 2.

Guía de práctica № 1

\begin{tabular}{|c|c|c|c|}
\hline $\begin{array}{l}\text { looge } \\
\text { dole } \\
\text { Cologio }\end{array}$ & \multicolumn{2}{|c|}{$\begin{array}{l}\text { NOMBRE DE LA INSTITUCIÓN EDUCATIVA } \\
\text { Guía de practica } N^{\circ} 1\end{array}$} & \multirow{2}{*}{ 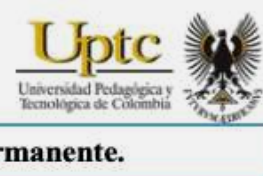 } \\
\hline $\bar{\square}$ & TítULO & Mando por impulso permanente. & \\
\hline $\bar{\square}$ & ASIGNATURA & \multicolumn{2}{|c|}{ Automatismos eléctricos. } \\
\hline$\square$ & TIEMPO & \multicolumn{2}{|c|}{ Tres (3) horas aprox. } \\
\hline Ti & LUGAR & \multicolumn{2}{|c|}{ Laboratorio de electricidad. } \\
\hline OB. & IVO GENERAL & & \\
\hline
\end{tabular}

En esta práctica se busca que el estudiante conozca, comprenda y monte en el tablero, un sistema de mando por impulso permanente, además se busca promover el uso de un software para simular tal esquema (Automation Studio).

Q OBJETIVOS ESPECÍFICOS:

$>$ Elaborar y comprobar el esquema de mando usando Automation Studio. 


\section{LA ENSEÑANZA

$>$ Analizar e interpretar el ciclo de funcionamiento.

$>$ Analizar e interpretar el esquema de interconexiones.

$>$ Realizar el montaje en el tablero y comprobar su funcionamiento.

\section{CONVENCIONES:}

En la siguiente tabla se ilustra el símbolo, la marca, los índices de los dispositivos que se emplearán en el desarrollo de esta guía de práctica.

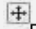

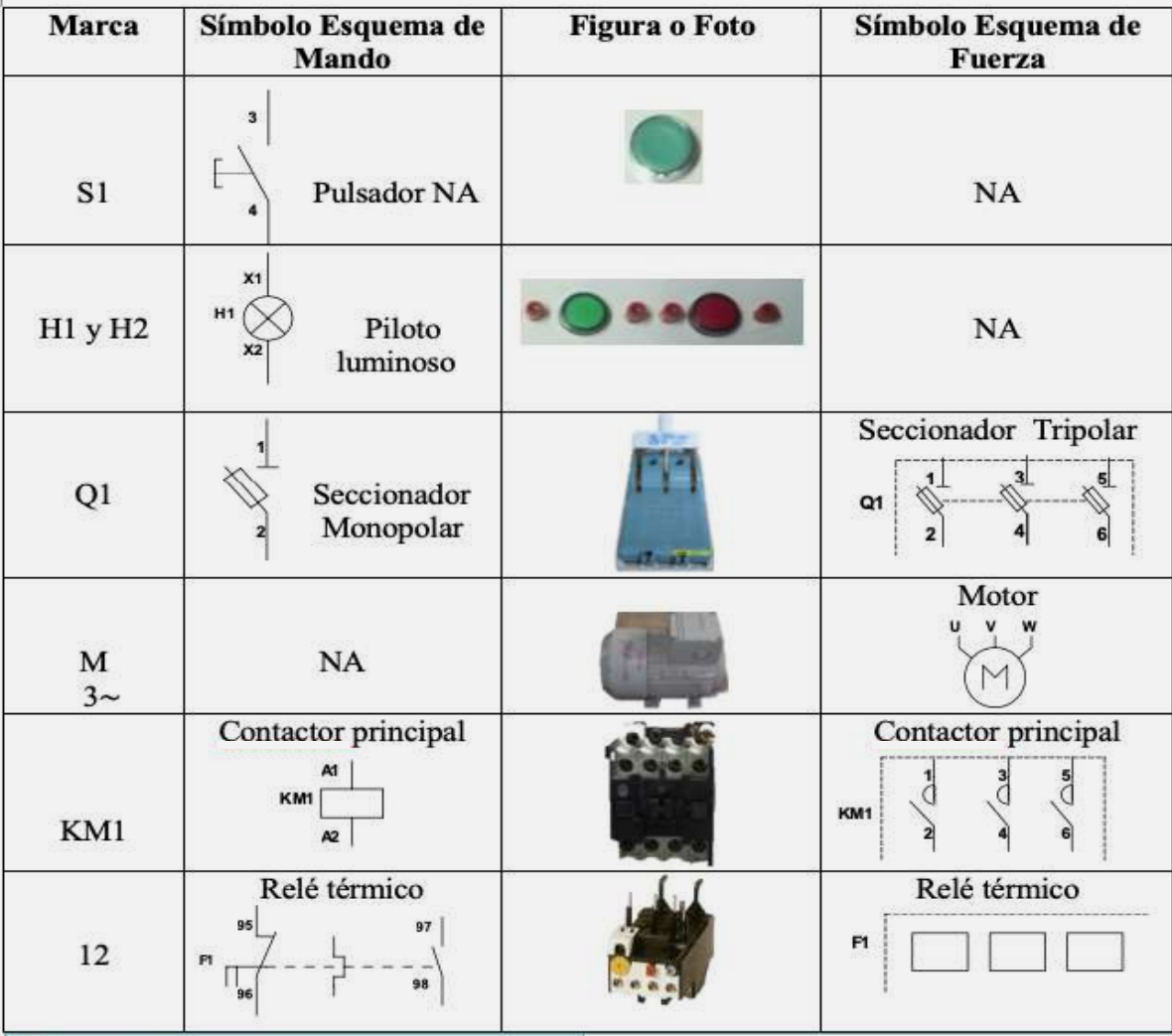

SIMULACION:

Elabore en Automation Studio los esquemas de mando y fuerza mostrados a continuación, y verifique su funcionamiento con la asesoría del profesor.

\section{ESQUEMAS:}


(L1)

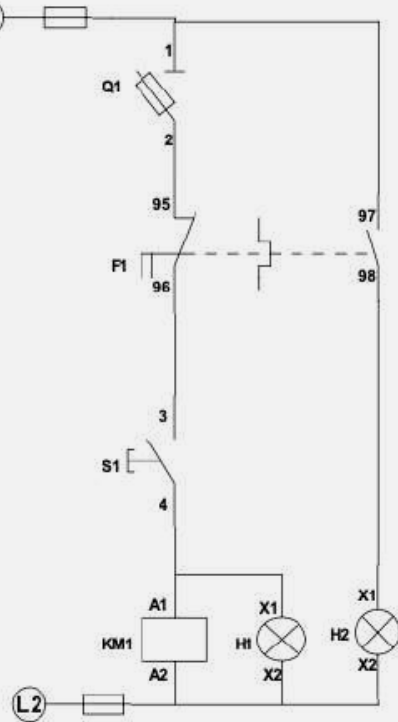

Esquema de mando

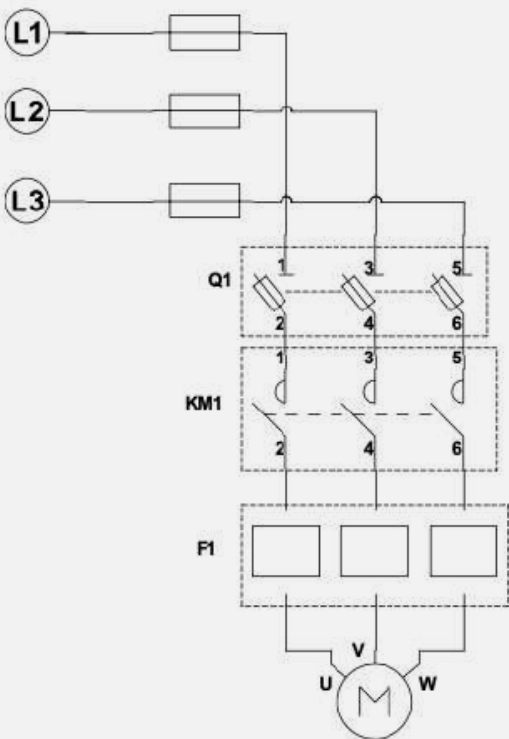

Esquema de fuerza

\section{() CICLO DE FUNCIONAMIENTO:}

El ciclo de funcionamiento describe paso a paso lo que ocurre con los dispositivos de mando, maniobra, señalización y protección en un automatismo eléctrico y es un importante referente para comprender el esquema de mando y el montaje que se haga en el tablero de automatismos.

Al pulsar S1 se cierra el circuito, la bobina de KM1 se energiza y el piloto luminoso $\mathrm{H} 1$ se enciende. Al energizarse la bobina de KM1 se cierran los contactos principales de KM1, poniéndose inmediatamente en marcha la carga. Si se deja de pulsar S1 se abre nuevamente el circuito, desenergizándose la bobina de KM1 y apagándose el piloto luminoso $\mathrm{H} 1$, por lo cual se abren nuevamente los contactos principales de KM1 deteniéndose la carga.

\section{$\square$ ESQUEMA DE INTERCONEXIONES:}

Basado en el esquema de mando, el esquema de interconexiones ilustra cómo se conectan los dispositivos de mando, maniobra, señalización y protección. Observe detenidamente el esquema de interconexiones y compárelo con el de mando.

\section{MATERIALES Y}

HERRAMIENTAS:

A continuación, se enuncian los materiales y herramientas requeridos para realizar el montaje en el tablero de automatismos.

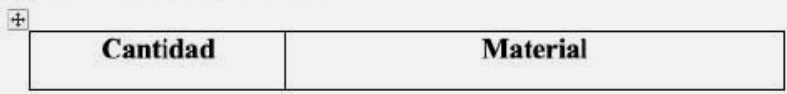




\begin{tabular}{|c|l|}
\hline 1 & Multímetro \\
\hline 2 & Destornilladores tipo estrella y pala \\
\hline 1 & Alicate \\
\hline $10 \mathrm{~m}$ & Cables de conexión $\mathrm{N}^{\circ} 16$ \\
\hline 2 & Bisturí o pinzas pelacables \\
\hline
\end{tabular}

\section{MONTAJE:}

Usando un marcador borrable identifique los dispositivos sobre el tablero en concordancia con el esquema de mando y fuerza.

Empleando el esquema de interconexiones como referente, monte el esquema de mando en el tablero. Una vez realizado el montaje, el docente lo revisará y autorizará que sea energizado.

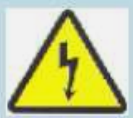

\section{ATENCION: NO DEBE ENERGIZAR EL SISTEMA SIN LA AUTORIZACION DEL PROFESOR.}

En caso de que no funcione el montaje abra el seccionador, desconecte la clavija trifásica de la toma respectiva, luego mediante el multímetro verifique continuidad. Iniciando con los terminales del seccionador y siguiendo con los dispositivos aguas abajo de este.

Si no es un problema de continuidad, revise las conexiones ya que puede ser posible que algún conductor no haya quedado convenientemente conectado.

\section{CUESTIONARIO:}

$>$ ¿Con sus propias palabras describa que es un sistema de mando por impulso permanente?

$>$ En el esquema de mando, ¿cuál es el propósito del piloto luminoso $\mathrm{H} 2$ ?

$>$ ¿En caso de que hubiese un cortocircuito que cree que debería suceder? Descríbalo con sus propias palabras.

$>$ ¿En caso de que hubiese una sobrecarga (sobrecorriente) que cree que debería suceder? Descríbalo con sus propias palabras.

¿Qué aplicación le daría a un sistema de mando por impulso permanente? de un ejemplo. $\square$ INFORME GUIA DE PRACTICA:

El informe de la guía de práctica debe incluir los siguientes elementos:

\section{Respuestas al cuestionario.}

$>$ Elabore un ensayo que describa la experiencia vivida.

$>$ Otros aspectos que el profesor estime convenientes.

\section{OBSERVACIONES:}

En esta guía de práctica se incluye el ciclo de funcionamiento y el esquema de interconexiones de forma que pueda tener un referente para elaborarlo en las demás prácticas.

\section{BIBLIOGRAFIA:}


Flower Leiva, Luis. Controles y Automatismos Eléctricos, séptima edición, Panamericana formas e impresos S.A 2001

CEMBRANOS NISTAL, Jesús. Automatismos eléctricos, neumáticos e hidráulicos.

SCHNEIDER Manual electrotécnico.

ODINFOGRAFIA:

http://www.electroindustria.com/nuevo/aplicacion_info.asp?id=1082

http://www.isftic.mepsyd.es/w3/recursos/fp/cacel/CACEL1/menu_1.htm

ESQUEMA DE INTERCONEXIONES

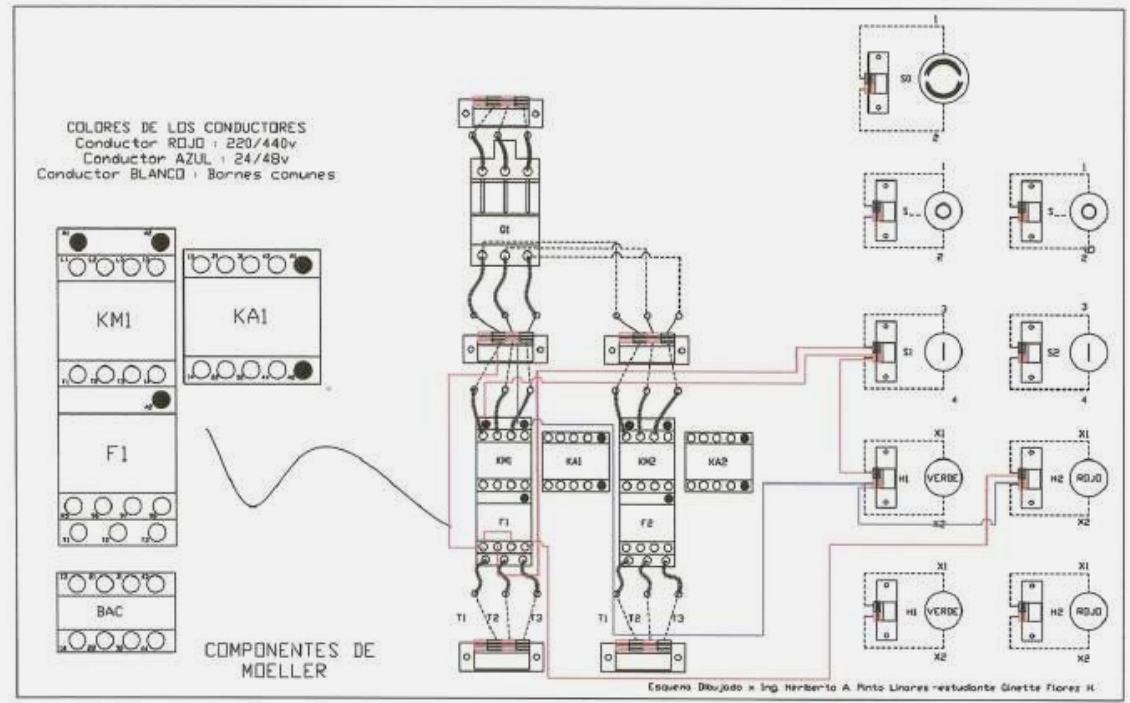

